\title{
Cochlear implant and tinnitus: a review
}

\author{
Santosh Kumar Swain* \\ Department of Otorhinolaryngology and Head and Neck Surgery, IMS and SUM hospital, Siksha "O” Anusandhan \\ University, Kalinga Nagar, Bhubaneswar, Odisha, India
}

Received: 22 October 2021

Accepted: 11 November 2021

\section{*Correspondence:}

Dr. Santosh Kumar Swain,

E-mail: santoshvoltaire@yahoo.co.in

Copyright: ( ) the author(s), publisher and licensee Medip Academy. This is an open-access article distributed under the terms of the Creative Commons Attribution Non-Commercial License, which permits unrestricted non-commercial use, distribution, and reproduction in any medium, provided the original work is properly cited.

\begin{abstract}
Tinnitus is the perception of a sound without any external auditory input and patient experiences as a ringing or buzzing sound in the ear or head. Tinnitus is a common and troublesome clinical entity that has existed for centuries. However, the exact etiology for tinnitus is not known. Electrical suppression of the tinnitus by cochlear implant is a secondary benefit to many cochlear implant recipients. Cochlear implants are often helpful to improve speech perception for those suffered with severe to profound hearing loss where hearing aids are no longer beneficial. There is high prevalence of tinnitus among patients with profound hearing loss which often reduced after cochlear implant. Although only few cochlear implant recipients show complete elimination of tinnitus after implantation and many uses result in improvement or stabilization. Cochlear implant causes appearance of homolateral tinnitus in small percentage of the cases. However, none of the cases of tinnitus which appear after cochlear implantation are thought to be severe to the patient. All literatures of cochlear implant and tinnitus were identified via Scopus, Google scholar, Medline and PubMed and analysed individually. Articles of cochlear implant and tinnitus included according to specified eligibility criteria. The review article analysed case series, case reports and original research on cochlear implant and tinnitus published in the English language. The aim of this review article is to provide a comprehensive review of the cochlear implant and its role in tinnitus.
\end{abstract}

Keywords: Cochlear implant, Tinnitus, Profound hearing loss, Sensorineural hearing loss

\section{INTRODUCTION}

Tinnitus is spontaneous, disagreeable auditory sensation, of varied origin with multiple etiologies. ${ }^{1}$ Tinnitus aurium means ringing of the ears and it can be classified into objective and subjective tinnitus. ${ }^{2}$ Objective tinnitus is defined as a sound which originates from the acoustic source within the patient and audible to the examiner whereas the subjective tinnitus has no acoustic tinnitus and the subjective tinnitus accounts for $95 \%$ of all types of tinnitus. ${ }^{3}$ Tinnitus is a common symptom found among patients with bilateral profound sensorineural hearing loss. Currently cochlear implant is a standard treatment for profound sensorineural hearing loss, and reportedly can minimize the tinnitus. ${ }^{4}$ This indication of cochlear implant for hearing loss and incapacitating tinnitus probably requires further convincing data on hypothesis that cochlear implant has impact on tinnitus magnitude and annoyance also in patients with unilateral deafness. It is also unclear whether the psychological and physical changes which accompany aging process influence the relief of the tinnitus after cochlear implant. Unilateral cochlear implantation is commonly performed in patients with bilateral profound sensorineural hearing loss. The additional benefit of this treatment is the reduction of subjective tinnitus. ${ }^{5}$ Interestingly, the sole cochlear implantation surgery itself may have positive impact on tinnitus. $^{6}$ It seems reasonable to suggest that positive expectations of patients with cochlear implants may have role in improvement of tinnitus. Study is also presenting with increasing tinnitus occurrence in $0 \%$ to $25 \%$ patients. ${ }^{7}$ The development of newly induced tinnitus following cochlear implantation ranges from $0 \%$ to $10 \%$ among patients without preoperative tinnitus. ${ }^{8}$ Because of 
increase of tinnitus and newly induced tinnitus following cochlear implant, a positive impact of cochlear implantation on the individual patient presenting or experiencing tinnitus cannot be predicted for certain. There are not much research concerning cochlear implantations and the effect on tinnitus. This review article will discuss on epidemiology, pathophysiology, cochlear implant surgery and tinnitus, severity of tinnitus, evaluation of tinnitus and current treatment of newly induced tinnitus after cochlear implantation.

\section{METHODS OF LITERATURE SEARCH}

Multiple systematic methods were used to find current research publications on cochlear implant and tinnitus. We started by searching the Scopus, PubMed, Medline, and Google Scholar databases online. A search strategy using PRISMA (Preferred reporting items for systematic reviews and meta-analysis) guidelines was developed. This search strategy recognized the abstracts of published articles, while other research articles were discovered manually from the citations. Randomized controlled studies, observational studies, comparative studies, case series, and case reports were evaluated for eligibility. There were total numbers of articles 94 (32 case reports; 34 cases series; 28 original articles) (Figure 1). This paper focuses only on tinnitus following cochlear implantation. This paper examines the epidemiology, pathophysiology, tinnitus following cochlear implant surgery, evaluation of severity of tinnitus and treatment of pre-cochlear implant tinnitus. This analysis provides a foundation for future prospective trials in cochlear implant and tinnitus. It will also serve as a catalyst for additional study into cochlear implant and its relation to tinnitus.

94 abstracts identified through PubMed, Scopus, Google Scholar and Medline database with the word cochlear implant and tinnitus 94 (32 case reports; 34 cases series; 28 original articles).

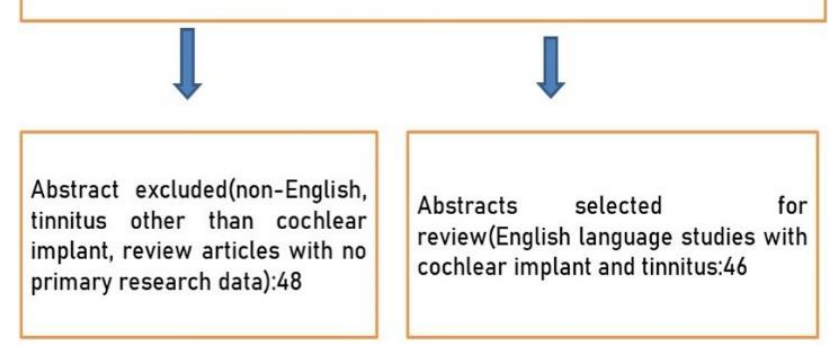

Figure 1: Method of literature search.

\section{HISTORY}

Jastreboff's neurophysiological model suggested the importance of the interaction of central auditory system, limbic system and the sympathetic autonomic system. ${ }^{9}$ The therapeutic effect of cochlear implant in preoperatively existing tinnitus had already been documented in the history of cochlear implant. In House and Brackmann documented that patient with cochlear implants get improvement of the previously existing tinnitus. ${ }^{10}$ This history suggests that possibility of using cochlear implantation help patients suffering from severe tinnitus. ${ }^{10}$ At one time or another, approximately $35 \%$ of the general population has suffered from tinnitus. In approximately $5 \%$ of the population, tinnitus is responsible for difficulties in falling asleep or shows a moderate to intense problem. Approximately $0.5 \%$ to $1 \%$ of the population experiences tinnitus which severely affects the routine life. ${ }^{11}$ As per this model, peripheral lesions generate an electrical signal, added to an efficient control of the encephalic stem system and to behavioral and emotional reactions, results in an unpleasant ringing or buzzing sensation.

\section{EPIDEMIOLOGY}

Tinnitus is a highly disturbing clinical entity, with high prevalence in sensorineural hearing loss patients. ${ }^{12}$ Subjective tinnitus has prevalence of $8 \%$ to $15 \%$ in general adult population. ${ }^{13}$ One study estimated the prevalence of adults with chronic tinnitus varies between $6 \%$ to $20 \% .^{13}$ The prevalence of tinnitus among bilateral profound sensorineural hearing loss is $65 \%$ to $100 \%$ with mean of approximately $80 \% .{ }^{14}$ Several studies seem to confirm the clinical findings that cochlear implant has a marked suppressive effect on tinnitus in majority of cochlear implant users. However, there are some uncertainties regarding to the prevalence and severity of tinnitus in profound sensorineural hearing loss patients, as well as suppression of tinnitus after cochlear implant. The prevalence of tinnitus in cochlear implant candidates ranges from $67 \%$ to $86 \% .^{5}$ One study showed that total tinnitus suppression rates vary from $2 \%$ to $83 \% .^{15}$ However, an increase of existing tinnitus ranges from $2 \%$ to $9 \%$, as well as a new onset of tinnitus. ${ }^{15}$ The induction of tinnitus found in $1 \%$ to $5 \%$ of patients as per recent study. ${ }^{16}$ Around $1 \%$ to $3 \%$ suffered disabling tinnitus interferes with their quality of life.

\section{PATHOPHYSIOLOGY}

The exact pathophysiology of the tinnitus is not known. Most of the hypothesis state that tinnitus occurs due to maladaptive plastic changes in the central auditory pathways as a reaction to the peripheral hearing loss. ${ }^{17}$ Recent studies demonstrated that occurrence of the neuronal plasticity and cortical reorganization in relation to perception of sound after injury to the cochlea. ${ }^{18}$ The disproportional increase in number of neurons sensitive to remaining frequencies of hearing or greater spontaneous activity in organized locations may explain the tinnitus sensation. The high prevalence of tinnitus is consistent with the fact that individuals with hearing loss are more likely to suffer from tinnitus. ${ }^{19}$ Reduction of the tinnitus after cochlear implants is better explained by several mechanisms like acoustic masking, habituation, direct stimulation of the auditory nerve and reorganization of the cortical areas. ${ }^{20}$ Tinnitus is considered 
to be the result of changes in neural activity in the auditory pathway and auditory cortical area of the brain, caused by decreased or lack of auditory input, for instance due to hearing loss. ${ }^{21}$ More than $60 \%$ of patients with severe to profound hearing loss have tinnitus. ${ }^{22}$ Although more research are needed, cochlear implantation is an effective technique for treatment of disabling tinnitus. Most probably six months use of cochlear implant might be enough to produce cerebral plastic reorganization which is able to minimize the tinnitus loudness. To improve the speech perception in patients with profound hearing loss, cochlear implant is an effective tool. ${ }^{23}$ This treatment by cochlear implant may alter the tinnitus related changes in neural activity in the auditory pathway and so diminishes the tinnitus complaints. $^{24}$

\section{TINNITUS AND COCHLEAR IMPLANT SURGERY}

Study suggested that electrical promontory stimulation is effective in suppression of the tinnitus. ${ }^{25}$ Reduced tinnitus has been documented after cochlear implant surgery. ${ }^{25}$ The insertion of electrode of cochlear implant is potentially a trauma to the remaining functional components of cochlea and thought to affect both necrotic and apoptic mechanisms of cell death and such insertion of electrode may influence the tinnitus among cochlear implant patients. ${ }^{26}$ However, there may be improvement of tinnitus with electrode insertion which described by some research papers, though the positive expectations of the patients in relation to implant may be a factor. ${ }^{27,28}$ There are few reports in patients with cochlear implant where electrode insertion exacerbates the tinnitus $(>10 \%)$ or aggravates it being heard for the first time $(>4 \%) .{ }^{29,30}$ There are also changes in tinnitus pitch and timber in cochlear patients. ${ }^{31}$ The post-surgical cochlear implant provides benefits in terms of tinnitus which raises few questions such as cochlear implant surgery justifiable for treatment of tinnitus. Similarly, the influence of tinnitus according to choose of ear for cochlear implant is also a complex subject. Some suggests that cochlear implant in the ear where tinnitus $\mathrm{s}$ more severe has chance for greater suppression. ${ }^{32}$

\section{EVALUATION OF SEVERITY OF TINNITUS}

Tinnitus handicap questionnaire (THQ) is used to measure the perception of physical and psychological well-being in tinnitus patients. It consists of 27 questions classified into three categories such as social, emotional and behavioral tinnitus effects; tinnitus and hearing; and outlook on tinnitus. Patients respond whether or not they agreed with statements on a scale of 0 to 100.Total scores are calculated per category, after which the mean value of THQ is calculated. ${ }^{33}$ Tinnitus handicap inventory (THI) questionnaires consist of 25 questions for influence of tinnitus on activities of daily life. ${ }^{34}$ There are 11 questions for functional effects on tinnitus, 9 questions for emotional responses to tinnitus, and 5 questions for catastrophic responses to tinnitus. Here, every 'yes' counts for 4 points, every 'sometimes' for 2 points and every 'never 'for 0 points. There is total score between 0 and 100. Scores less than 16 indicate slight handicap, scores from 18 to 38 indicate mild tinnitus, scores from 38 to 56 correspond to severe tinnitus, and scores more than 78 indicate catastrophic tinnitus. One study showed tinnitus improvement after six months with CI fitting and $36 \%$ reported total loudness suppression and another $41 \%$ reported a reduction; annoyance scores reduced in $75 \%$ of patients and $72.2 \%$ reported these benefits in the THI questionnaire. ${ }^{35}$ Tinnitus significantly affect the quality of life and may cause stress, depression, anxiety and may result in suicidal tendencies in some patients. ${ }^{36}$ Reports have been documented the possibility of tinnitus worsening or being exacerbated following cochlear implant surgery. ${ }^{37}$ Few patients developed postsurgical onset of tinnitus and labyrinthine symptoms when the implant is activated. ${ }^{38}$ The probable explanation for this tinnitus us that program adjustment is not happened owing to the short period of activation.

\section{TREATMENT OF PRE-COCHLEAR IMPLANT TINNITUS}

Many patients those with sensorineural hearing loss present with tinnitus, particularly those with an indication for cochlear implant. ${ }^{39}$ Although several treatment options have been recommended for managing tinnitus, none of them has been total effective for abolishing the tinnitus. $^{40}$ If the tinnitus disturbs the sleep i.e., troublesome tinnitus may need programming of cochlear implant device for night time use. There are two options: first one utilizes a program which facilitates night time use of an environmental sound like wind, rain or ocean sound and this is effective as tinnitus therapy in persons with hearing. ${ }^{41}$ The second option considers an implant electrode configuration which provides barely suprathreshold stimulation in night to decrease the awareness of the tinnitus. As in hearing aids, optimization of the cochlear implant for tinnitus may involve programming which decreases speech discrimination abilities. The tinnitus program should be addition to the program which is in general use of the cochlear implant (speech communication). There is an ongoing worldwide discussion on whether or not bilateral cochlear implantation should be standard care towards bilateral severe to profound deafness. Contrary to unilateral cochlear implantation, however, study reported on the impact of bilateral cochlear implantation on tinnitus where the bilateral cochlear implantation has positive effect on preoperative tinnitus. ${ }^{42}$ In sequential group of patients for cochlear implantation, the additional benefit of the second cochlear implant is total suppression of tinnitus in comparison to unilateral situation.

\section{POST-COCHLEAR IMPLANT TINNITUS}

Tinnitus is a common occurrence in pediatric age group those undergo cochlear implantation. ${ }^{43}$ The higher 
prevalence of tinnitus is in patients with cochlear implantation than non-implanted children with profound hearing loss. ${ }^{43}$ There was no evidence that prevalence of tinnitus was influenced by hearing loss, time elapsed since implantation and age at cochlear implantation. In children with bilateral cochlear implants, there is increased evidence of tinnitus in those with a longer inter-implant delay than those with a short delay or simultaneous implantation. ${ }^{43}$ Although the high tinnitus prevalence, quality of life is less affected with a small proportion of pediatric patients reporting sleep disturbances. Prevalence of tinnitus among children with normal and impaired hearing are given in Table $1 .{ }^{44-48}$

Table 1: Prevalence of tinnitus among children with normal and impaired hearing.

\begin{tabular}{|c|c|c|c|}
\hline Author & $\begin{array}{l}\text { Normal } \\
\text { hearing } \\
\text { children } \\
\text { with } \\
\text { tinnitus } \\
(\%)\end{array}$ & $\begin{array}{l}\text { Impaired } \\
\text { hearing } \\
\text { children } \\
\text { with } \\
\text { tinnitus } \\
(\%)\end{array}$ & Annoyance \\
\hline Holgers et al $^{44}$ & 13 & 9 & - \\
\hline Holgers et al $^{45}$ & 53 & - & 27 \\
\hline Aksoy et al ${ }^{46}$ & 9 & - & 6 \\
\hline Coelho et al $^{47}$ & 39 & 44 & 20 \\
\hline Martin et al $^{48}$ & 50 & 50 & - \\
\hline
\end{tabular}

\section{CONCLUSION}

Tinnitus is a common clinical problem associated with cochlear implant candidates and cochlear implant users. Cochlear implant is often helpful to reduce tinnitus and tinnitus handicap. A short duration of tinnitus before cochlear implantation, a tonal tinnitus, a higher tinnitus handicap prior to implantation, and a round window insertion surgical technique correlate with an optimistic influence of cochlear implantation on the tinnitus. Although cochlear implant improves or stabilize the tinnitus perception in most of adult cochlear implant receivers, only rarely does total elimination of the tinnitus. The variable effect of cochlear implant on tinnitus remains unexplained. This may be due to absence of residual inhabitation of tinnitus in patient using multichannel cochlear implants. Further research is required in larger and a more uniform study population in regards to hearing abilities, type of tinnitus (unilateral/tonal) and age of the patients.

\section{Funding: No funding sources}

Conflict of interest: None declared

Ethical approval: Not required

\section{REFERENCES}

1. Swain SK, Nayak S, Ravan JR, Sahu MC. Tinnitus and its current treatment-Still an enigma in medicine. Journal of the Formosan Medical Association. 2016;115(3):139-44.
2. Swain SK, Behera IC, Sahu MC. Tinnitus among children-Our experiences in a tertiary care teaching hospital of eastern India. Pediatria Polska. 2017;92(5):513-7.

3. Erlandsson S, Dauman N. Categorization of tinnitus in view of history and medical discourse. International journal of qualitative studies on health and well-being. 2013;8:23530.

4. Andersson G, Freijd A, Baguley DM, Idrizbegovic E. Tinnitus distress, anxiety, depression, and hearing problems among cochlear implant patients with tinnitus. J Am Acad Audiol. 2009; 20:315-9.

5. Quaranta N, Wagstaff S, Baguley DM. Tinnitus and cochlear implantation. Int J Audiol. 2004;43:245-51.

6. Greimel KV, Meco C, Mair A, Kohlbo“ck G, Albegger K. How is tinnitus influenced by cochlear implantation? HNO. 2003;51:226- 31.

7. Amoodi HA, Mick PT, Shipp DB, Friesen LM, Nedzelski JM, Chen JM et al. The effects of unilateral cochlear implantation on the tinnitus handicap inventory and the influence on quality of life. Laryngoscope. 2011;121(7):1536-40.

8. Kompis M, Pelizzone M, Dillier N, Allum J, DeMin N, Senn P. Tinnitus before and 6 months after cochlear implantation. Audiol Neurootol. 2012;17:161-8.

9. Jastreboff PJ. Phantom auditory perception (tinnitus): Mechanism of generation and perception. Neurosci Res. 1990;8:221-54.

10. House JW, Brackmann DE. Tinnitus: surgical treatment. Ciba Found Symp. 1981;85:204-16.

11. McCombe A, Baguley D, Coles R, McKenna L, McKinney C, Windle-Taylor P. British Association of Otolaryngologists, Head and Neck Surgeons. Guidelines for the grading of tinnitus severity: the results of a working group commissioned by the British Association of Otolaryngologists, Head and Neck Surgeons, 1999. Clin Otolaryngol Allied Sci. 2001;26:388-93.

12. Swain SK, Sahu MC, Choudhury J. Sudden sensorineural hearing loss in children: Our experiences in tertiary care teaching hospital of eastern India. Pediatria Polska-Polish J Paediatr. 2018;93(2):127-31.

13. Langguth B, Kreuzer PM, Kleinjung T, De Ridder D. Tinnitus: causes and clinical management. Lancet Neurol. 2013;12(9):920-30.

14. Baguley DM, Atlas MD. Cochlear implants and tinnitus. Prog Brain Res. 2007;166:347-55.

15. Quaranta N, Wagstaff S, Baguley DM. Tinnitus and cochlear implantation. Int J Audiol. 2004;43:245-51.

16. Jeppesen J, Faber CE. Surgical complications following cochlear implantation in adults based on a proposed reporting consensus. Acta Otolaryngol. 2013;133:1012-21.

17. Swain SK. Hearing loss, tinnitus and vertigo among pediatric patients with COVID-19 infections: a review. Int J contemporary Pediatr. 2021;8(10):175661. 
18. Swain SK, Das A, Sahu MC, Das R. Neonatal hearing screening: Our experiences at a tertiary care teaching hospital of eastern India. Pediatria Polska. 2017;92(6):711-5.

19. Han BI, Lee HW, Kim TY, Lim JS, Shin KS. Tinnitus: characteristics, causes, mechanisms, and treatments. J Clin Neurol. 2009;5(1):11-9.

20. Swain SK, Achary S, Das SR. Vertigo in pediatric age: Often challenge to clinicians. Int J Cur Res Rev. 2020;12(18):136-41.

21. Ramakers GG, Van Zon A, Stegeman I, Grolman W. The effect of cochlear implantation on tinnitus in patients with bilateral hearing loss: a systematic review. Laryngoscope. 2015;125(11):2584-92.

22. Kim DK, Bae SC, Park KH, Jun BC, Lee DH, Yeo $\mathrm{SW}$ et al. Tinnitus in patients with profound hearing loss and the effect of cochlear implantation. Euro Arch Oto-Rhino-Laryngol. 2013;270(6):1803-8.

23. Swain SK, Pattnaik T, Mohanty JN. Otological and rhinological manifestations in pregnancy: Our experiences at a tertiary care teaching hospital of East India. Int J Health Allied Sci. 2020;9(2):159-63.

24. Dixon PR, Crowson M, Shipp D, Smilsky K, Lin VY, Le T et al. Predicting reduced tinnitus burden after cochlear implantation in adults. Otol Neurotol. 2020;41(2):196-201.

25. Quaranta N, Wagstaff S, Baguley D. Tinnitus and cochlear implantation. Int J Audiol. 2004;43:245-51.

26. Adunka O, Kiefer J. Impact of electrode insertion depth on intracochlear trauma. Otolaryngol head neck surg. 2006;135(3):374-82.

27. Sahoo L, Swain SK, Das A, Nahak B, Munjal S. Clinical concerns of hearing loss in old age: an Indian perspective. J Geriatr Care Res. 2020;7(2):5663.

28. Greimel KV, Meco C, Kohlbock F, Mair A, Albegger K. Changes in tinnitus after cochlear implantation. InProceedings of the seventh international tinnitus seminar. 2002;6-10.

29. Gibson WP, Aran JM, Dauman R. The effect of electrical stimulation and cochlear implantation on tinnitus. In Tinnitus 91: Proceedings of the Fourth International Tinnitus Seminar. 1992;403-8.

30. Miyamoto RT, Bichey BG. Cochlear implantation for tinnitus suppression. Otolaryngol Clin North Am. 2003;36(2):345-52.

31. Souliere CR, Kileny PR, Zwolan TA, Kemink JL. Tinnitus suppression following cochlear implantation: A multifactorial investigation. Arch Otolaryngol head Neck Surg. 1992;118(12):1291-7.

32. Demajumdar R, Stoddart R, Donaldson I, Props W. Tinnitus, cochlear implants and how they affect patients. J Laryngol Otol Suppl. 1999;113:24-6.

33. Kuk FK, Tyler RS, Russell D, Jordan H. The psychometric properties of a tinnitus handicap questionnaire. Ear Hear. 1990;11(6):434-45.

34. Newman CW, Jacobson GP, Spitzer JB. Development of the Tinnitus Handicap Inventory.
Arch Otolaryngol Head Neck Surg. 1996;122(2):143-8.

35. Bovo R, Ciorba A, Martini A. Tinnitus and cochlear implants. Auris Nasus Larynx. 2011;38(1):14-20.

36. Schaaf H, Eipp C, Deubner R, Hesse G, Vasa R, Gieler U. Psychosocial aspects of coping with tinnitus and psoriasis patients. A comparative study of suicidal tendencies, anxiety and depression. HNO. 2009;57(1):57-63.

37. Quaranta N, Wagstaff S, Baguley D. Tinnitus and cochlear implantation. Int J Audiol. 2004;43:245-51.

38. Swain SK, Anand N, Mishra S. Vertigo among elderly people: Current opinion. J Med Society. 2019;33(1):1-5.

39. Swain SK, Pati BK, Mohanty JN. Otological manifestations in pregnant women-A study at a tertiary care hospital of eastern India. J Otol. 2020;15(3):103-6.

40. Swain SK, Munjal S, Shajahan N. Vertigo in children: Our experiences at a tertiary care teaching hospital of eastern India. J Scientific Society. 2020;47(2):74-8.

41. Handscomb L. Use of bedside sound generators by patients with tinnitus-related sleeping difficulty: which sounds are preferred and why? Acta OtoLaryngologica. 2006;126(556):59-63.

42. van Zon A, Smulders YE, Ramakers GG, Stegeman I, Smit AL, Van Zanten GA et al. Effect of unilateral and simultaneous bilateral cochlear implantation on tinnitus: a randomized controlled trial. Laryngoscope. 2015;126(4):956-61.

43. Chadha NK, Gordon KA, James AL, Papsin BC. Tinnitus is prevalent in children with cochlear implants. Int $\mathrm{j}$ pediatric otorhinolaryngol. 2009;73(5):671-5.

44. Holgers KM, Pettersson B. Noise exposure and subjective hearing symptoms among school children in Sweden. Noise and health. 2005;7(27):27-37.

45. Aksoy S, Akdogan Ö, Gedikli Y, Belgin E. The extent and levels of tinnitus in children of central Ankara. Int $\mathrm{j}$ pediatric otorhinolaryngol. 2007;71(2):263-8.

46. Coelho CB, Sanchez TG, Tyler RS. Tinnitus in children and associated risk factors. Progress brain res. 2007;166:179-91.

47. Martin K, Snashall S. Children presenting with tinnitus: a retrospective study. Brit $\mathrm{j}$ audiol. 1994;28(2):111-5.

48. Holgers KM. Tinnitus in 7-year-old children. Eur $\mathrm{j}$ pediatrics. 2003;162(4):276-8.

Cite this article as: Swain SK. Cochlear implant and tinnitus: a review. Int J Otorhinolaryngol Head Neck Surg 2021;7:1960-4. 